\title{
Long term non-invasive domiciliary assisted ventilation for respiratory failure following thoracoplasty
}

\author{
Mark Jackson, Ian Smith, Martin King, John Shneerson
}

\begin{abstract}
Background - Ventilatory failure is a well recognised complication of patients who have had a thoracoplasty for tuberculosis, but there are few data regarding the value of long term non-invasive assisted ventilation in this situation.

Methods - Thirty two patients who had had a thoracoplasty $20-46$ years previously and who had developed respiratory failure were treated with nocturnal cuirass assisted ventilation or nasal positive pressure ventilation. Their survival and changes in arterial blood gases, nocturnal oximetry, and pulmonary function tests were assessed.
\end{abstract}

Results - The actuarial survival rates at one, three, five, and seven years after starting treatment were $91 \%, 74 \%, 64 \%$, and $55 \%$, respectively. Only seven of the 13 deaths were directly attributable to chronic respiratory or cardiac failure. The arterial $\mathrm{Po}_{2}, \mathbf{P C O}_{2}$, mean nocturnal oxygen saturation, vital capacity, and maximal inspiratory and expiratory pressures had all improved at the time of the initial posttreatment assessment (mean 12 days after starting treatment), but no subsequent improvements were seen after up to 48 months of follow up. Neither survival nor physiological improvements were correlated with the patients' age, the interval since thoracoplasty, or the pretreatment arterial blood gas tensions or results of pulmonary function tests.

Conclusions - These results show that, even when ventilatory failure has developed, the prognosis with non-invasive assisted ventilation is good and the physiological abnormalities can be partially reversed. Patients who develop respiratory failure after a thoracoplasty should be considered for this type of long term domiciliary treatment.

(Thorax 1994;49:915-919)

Treatment by surgical collapse, especially thoracoplasty, played an important part in the management of pulmonary tuberculosis before the advent of effective antituberculous chemotherapy. Thoracoplasty causes a restrictive defect as a consequence of pleural thickening, thoracic cage deformity, and secondary scoliosis. ${ }^{1-4}$ Airflow obstruction is also common in these patients and, when severe, is associated with hypoxia and hypercapnia..$^{5-7}$ Many patients who were treated with a thoracoplasty now have respiratory symptoms and some are at risk of developing respiratory failure. ${ }^{7}$ Domiciliary assisted ventilation is a well established treatment for respiratory failure in patients with neuromuscular and chest wall disorders, but there is little information about the outcome of this treatment in patients who have had a thoracoplasty. The aims of this study were to document the physiological results and survival of thoracoplasty patients who have been treated with assisted ventilation.

\section{Methods}

Thirty two patients who had undergone thoracoplasty were established on assisted ventilation at our centre between December 1983 and September 1993. There were eight men and 24 women of mean age $62 \cdot 3$ (range $35-78$ ) years. Sixteen had left thoracoplasties, 14 were right sided, and two had bilateral thoracoplasties. Most of the patients had also had an artificial pneumothorax performed on the contralateral side. All of the thoracoplasties were performed between 1947 and 1958 with the exception of one which was performed in 1971 in India. The mean age at time of thoracoplasty was 26 (range 15-46) years and the mean interval between thoracoplasty and start of assisted ventilation was $36 \cdot 6$ (range 20-46) years.

The indications for assisted ventilation included chronic stable but symptomatic ventilatory failure, evidence of progressively deteriorating ventilatory failure, and severe ventilatory failure during an apparently mild episode of upper or lower respiratory tract infection. The patients were receiving appropriate non-ventilatory management of their respiratory failure before starting long term domiciliary assisted ventilation.

Arterial oxygen $\left(\mathrm{PaO}_{2}\right)$ and carbon dioxide $\left(\mathrm{PaCO}_{2}\right)$ tensions were measured with the patient at rest and breathing room air and analysed in a Radiometer blood gas analyser. Forced expiratory volume in one second $\left(\mathrm{FEV}_{1}\right)$ and vital capacity (VC) were measured with a Vitalograph spirometer. Functional residual capacity (FRC) was measured by helium dilution (P K Morgan, Gillingham, UK) and total lung capacity (TLC) and residual volume (RV) were calculated. Maximum mouth pressures were recorded using a pressure transducer (Si-Plan Electronics Research Ltd, Stratford 
upon Avon, UK) connected to a mouthpiece with a 22 gauge leak. Maximum inspiratory pressure (MIP) was obtained at RV and maximum expiratory pressure (MEP) at TLC. Maximum voluntary ventilation was estimated by measuring expired volume whilst the patient breathed for 15 seconds through a low resistance valve ( $\mathrm{PK}$ Morgan). Predicted values for measurements of lung function were calculated for each subject using standard regression equations. ${ }^{8}$ Whenever possible the arterial gases and pulmonary function tests were measured before the start of assisted ventilation. These tests were repeated on each subsequent admission and the results that are presented are the values prior to treatment, shortly following the establishment of assisted ventilation (mean 12 days), and at $6,12,18$, 24, 36, and 48 months. Before 1988 only cuirass assisted ventilation using custom made cuirass shells ${ }^{9}$ with the Newmarket pump (SiPlan Electronics Research Ltd $)^{10}$ was available in our centre. Since that date patients have been treated with this or with time cycled nasal intermittent positive pressure ventilation (NIPPV) using the Monnal-D ventilator (Taema Ltd, Paris, France) with standard silicon masks (Respironics Ltd, Murrysville, USA). During the initial and subsequent admissions the ventilators were adjusted to ensure optimal ventilation as judged from repeated overnight studies during which oxygen saturation and transcutaneous carbon dioxide pressure were recorded. In all patients the domiciliary assisted ventilation was required during sleep only. Six patients required supplemental oxygen while using their assisted ventilation in order to achieve adequate nocturnal oxygenation. Compliance with treatment was assessed from the "hours used" clocks on the ventilators.

STATISTICS

Survival data were calculated using a life table

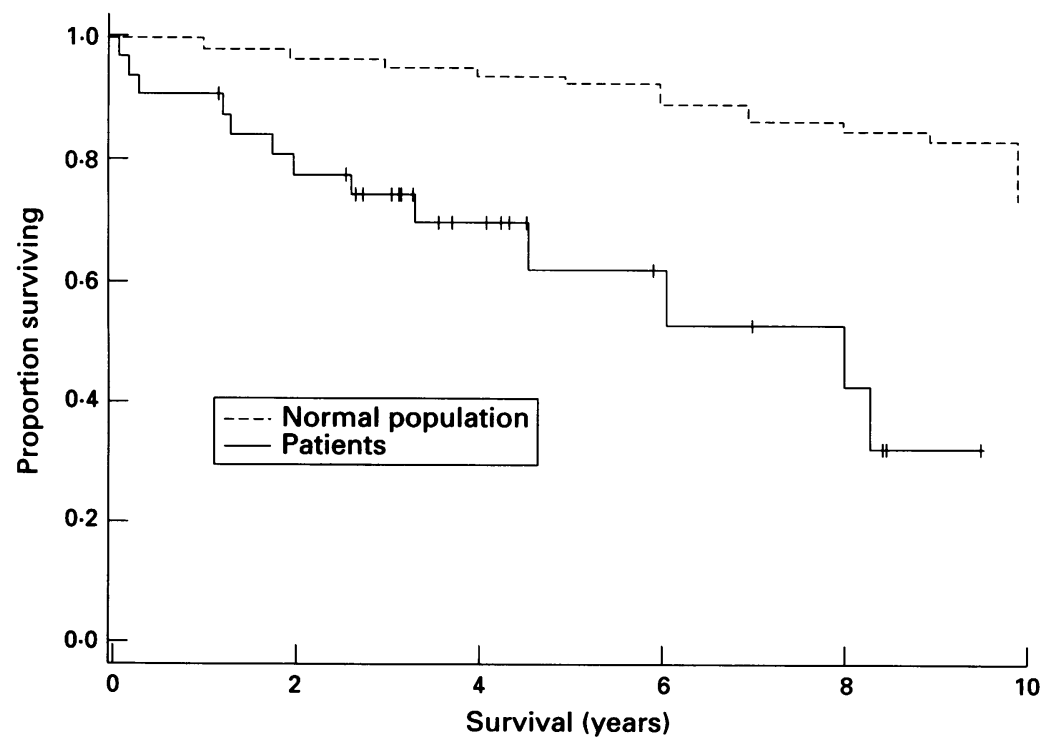

Figure 1 Actuarial survival of the 32 patients and an age and sex matched "normal" population. procedure. Comparison with an age and sex matched population derived from standard tables (OPCS Mortality Statistics 1991 (England and Wales)) was made using the log rank test.

Longitudinal comparison of physiological values was made using $t$ tests for paired samples. Statistical significance was assumed for $\mathrm{p}$ values less than 0.05 .

\section{Results}

Nineteen patients were initially established on cuirass ventilation but six of these subsequently changed to NIPPV. Three of these changed at between one and three months because of discomfort and limited improvement, one changed after 13 months because of loss of control of his hypoventilation following a respiratory tract infection, and two changed after 70 and 72 months - one because of the onset of dementia in order to try to improve compliance with treatment, and the other for social reasons. Thirteen patients were initially established on NIPPV and none have changed their mode of ventilation. Overall, compliance with treatment was good with the ventilators being used for a mean of $7 \cdot 4$ hours per day.

The actuarial survival of these patients is shown in fig 1 , along with a constructed survival curve of an age and sex matched "normal" population. The calculated cumulative survival rates at one, three, five, and seven years were $91 \%, 74 \%, 64 \%$, and $55 \%$, respectively. The excess mortality of the patients compared with the normal group is significant $(p<0.001)$.

Thirteen patients have died after a mean of $36 \cdot 7$ (range 1-100) months. One patient died of shock following rupture of eosophageal varices, one patient developed multi infarct dementia after six years of successful treatment and died of respiratory failure when she was unable to comply with treatment, one patient died in her local hospital, probably from a pulmonary embolus, following a repair of a fractured femur. Two patients did not comply with treatment adequately (using assisted ventilation on average for less than four hours per night) and died of respiratory failure. One patient developed a respiratory infection and died in her local hospital without appropriate agg-

Table 1 Arterial gases and pulmonary function tests before starting assisted ventilation

\begin{tabular}{|c|c|c|c|}
\hline & & $\begin{array}{l}95 \% \text { confidence } \\
\text { interval }\end{array}$ & $n$ \\
\hline $\mathrm{PaO}_{2}(\mathrm{kPa})$ & $7 \cdot 1$ & $6 \cdot 4$ to $7 \cdot 8$ & 29 \\
\hline $\mathrm{PaCO}_{2}(\mathrm{kPa})$ & $7 \cdot 8$ & $7 \cdot 26$ to 8.33 & 29 \\
\hline $\mathrm{FEV}_{1}$ (l) & 0.56 & 0.47 to 0.65 & 26 \\
\hline \%pred $\mathrm{FEV}_{1}$ & $24 \cdot 1$ & $20 \cdot 7$ to $27 \cdot 7$ & \\
\hline VC (l) & 1.06 & 0.89 to 1.23 & 26 \\
\hline \%pred VC & 37 & $32 \cdot 7$ to $41 \cdot 2$ & \\
\hline $\mathrm{FEV}_{1} / \mathrm{FVC}(\%)$ & $54 \cdot 9$ & 48.6 to $61 \cdot 1$ & 26 \\
\hline TLC (1) & $2 \cdot 58$ & 2 to 316 & 20 \\
\hline$\%$ pred TLC & 52 & 45.3 to 58.7 & \\
\hline FRC (1) & 1.65 & 1.22 to 2.07 & 20 \\
\hline \%pred FRC & $57 \cdot 9$ & 47.4 to 68.4 & \\
\hline $\mathrm{MIP}\left(\mathrm{cm} \mathrm{H}_{2} \mathrm{O}\right)$ & $38 \cdot 7$ & 27.5 to 49.8 & 16 \\
\hline $\mathrm{MEP}\left(\mathrm{cm} \mathrm{H}_{2} \mathrm{O}\right)$ & $67 \cdot 8$ & 46.4 to 89.2 & 16 \\
\hline $\mathrm{MVV}(1 / \mathrm{min})$ & $28 \cdot 2$ & 23 to 33.5 & 16 \\
\hline
\end{tabular}

$\%$ pred $=\%$ of predicted value; TLC $=$ total lung capacity, FRC = functional residual capacity, $M I P=$ maximum inspired $\mathrm{FRC}=$ functional residual capacity, $\mathrm{MIP}=$ maximum inspired
pressure, $\mathrm{MEP}=$ maximum expired pressure, $\mathrm{MVV}=$ maximum pressure, $M E P=\max$
voluntary ventilation 


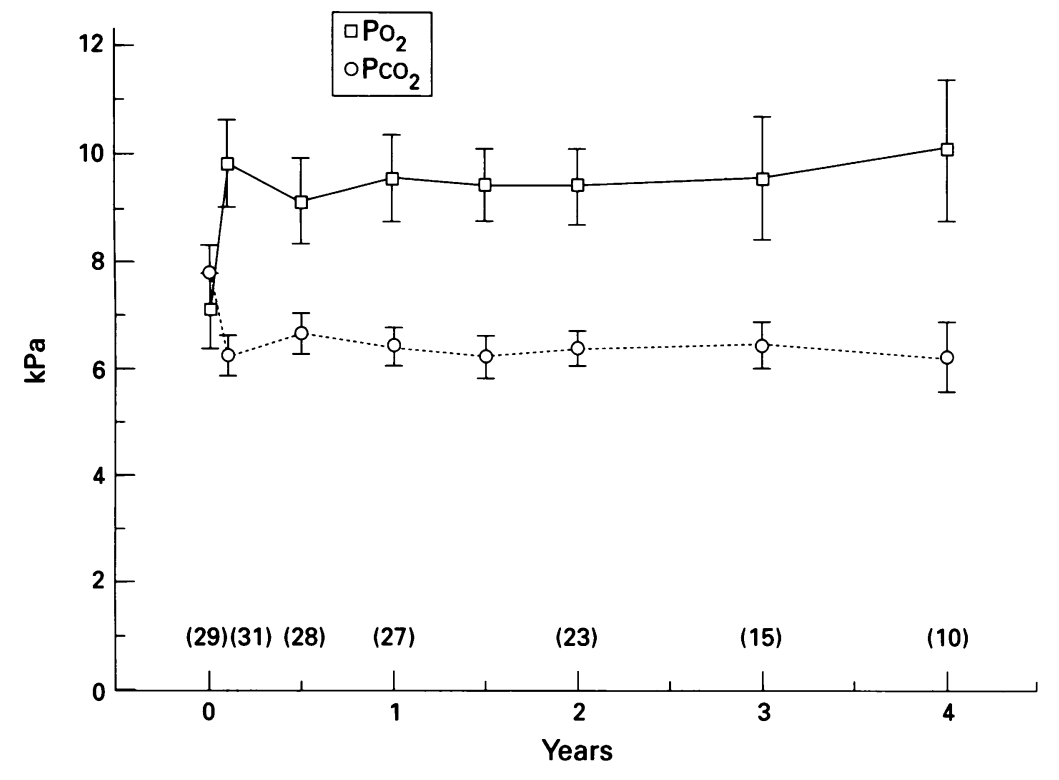

Figure 2 Changes in arterial gas tensions in $\mathrm{kPa}$ (mean and $95 \% \mathrm{CI}$ ) of all patients before ( 0 years) and after treatment with assisted ventilation. Figures in brackets indicate the number of patient values at each point.

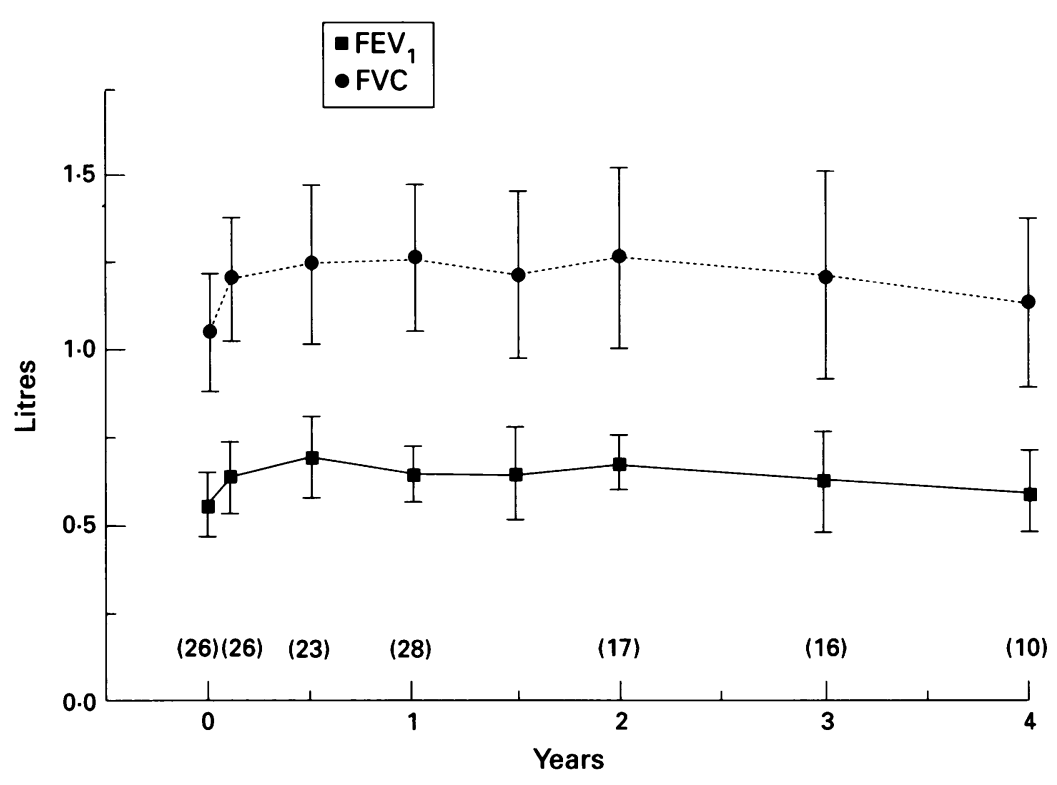

Figure 3 Changes in spirometric values (mean and 95\% CI) of all patients before (O years) and after treatment with assisted ventilation. Figures in brackets indicate the number of patient values at each point. ressive management. Seven patients died with progressively worsening arterial gases while awake with dependent oedema, raised venous pressure, and increasing diuretic requirements, and in four cases with ECG evidence of pulmonary hypertension despite regular assisted ventilation. Nineteen patients remain alive after a mean period of assisted ventilation of $51 \cdot 7$ (range 12-112) months.

Arterial gas values before assisted ventilation were not obtained in three patients who required emergency intubation in their referring hospitals. Spirometric testing could not be performed in six patients for similar reasons. In the remainder the pretreatment measurements of arterial gases and pulmonary function showed that the patients were profoundly hypoxic (mean $\mathrm{PaO}_{2} 7 \cdot 1 \mathrm{kPa}$ ) with carbon dioxide retention (mean $\mathrm{PaCO}_{2} 7 \cdot 8 \mathrm{kPa}$ ) and that they had a severe mixed restrictive and obstructive defect with a mean percentage predicted $\mathrm{FEV}_{1}$, VC, and TLC of $24 \cdot 1 \%, 37 \%$, and $52 \%$, respectively (table 1). The changes in arterial gases with assisted ventilation are shown in fig 2 .

Comparison of pretreatment with the immediate post-treatment measurements demonstrated significant improvements in both the $\mathrm{PaO}_{2}$ (mean paired difference $=2.54 ; 95 \% \mathrm{CI}$ 1.67 to $3.42 ; \mathrm{p}<0.001$ ) and the $\mathrm{PaCO}_{2}$ (mean paired difference $=1.53 ; 95 \%$ CI 0.96 to $2 \cdot 11$; $\mathrm{p}<0.001)$. There were no significant changes thereafter. Similarly, comparison of pulmonary function tests before and after treatment demonstrated a significant improvement in VC (mean paired difference 0.16 ; $95 \%$ CI 0.015 to $0.314 ; \mathrm{p}=0.033$ ) and MEP (mean paired difference $11.85 ; 95 \%$ CI 1.73 to $7.45 ; p=$ $0.025)$. An improvement was seen in the MIP between the pretreatment value and that at six months (mean paired difference $13.11 ; 95 \%$ CI 4.5 to $21.7 ; p=0.008)$. The changes in spirometric values are shown in fig 3 .

Complete pretreatment overnight oximetry studies were obtained in only 10 patients and these had profound hypoxaemia, spending a mean of $86 \%$ of the overnight period at or below an oxygen saturation of $90 \%$. The remaining patients were either tansferred to our centre having been intubated or required intervention before completion of an overnight study. The changes in the oxygen saturation during overnight studies following treatment with assisted ventilation in the patients who did not require supplemental overnight oxygen are shown in table 2 along with the mean daytime $\mathrm{Po}_{2}$ values of this group of patients.

There were no significant differences be-

Table 2 Summary of overnight oximetry studies, with the corresponding mean daytime $\mathrm{PO}_{2}$ values, in the patients who did not require supplemental overnight oxygen

\begin{tabular}{|c|c|c|c|c|c|c|c|c|}
\hline & $\begin{array}{l}\text { Before } \\
\text { treatment }\end{array}$ & $\begin{array}{l}\text { Before } \\
\text { discharge }\end{array}$ & 6 weeks & 6 months & 1 year & 2 years & 3 years & 4 years \\
\hline $\begin{array}{l}\text { Mean \% of overnight stuc } \\
\text { at or below } \mathrm{SaO}_{2} \text { of: } \\
90 \% \\
80 \% \\
70 \% \\
\text { Mean (SD) daytime } \mathrm{Po}_{2} \\
\text { No. of patients }\end{array}$ & $\begin{array}{l}86 \\
52 \\
23 \\
7 \cdot 5(1 \cdot 95) \\
10\end{array}$ & $\begin{array}{l}31 \\
3 \cdot 4 \\
0 \cdot 7 \\
10 \cdot 5(1 \cdot 64) \\
17\end{array}$ & $\begin{array}{l}34 \\
7 \cdot 5 \\
0 \cdot 6 \\
10 \cdot 2(1 \cdot 68) \\
16\end{array}$ & $\begin{array}{l}21 \\
0 \cdot 5 \\
0 \\
9 \cdot 9(1 \cdot 77) \\
14\end{array}$ & $\begin{array}{l}21 \\
1 \\
0 \\
10 \cdot 1(2) \\
15\end{array}$ & $\begin{array}{l}14 \\
0 \cdot 4 \\
0 \\
9 \cdot 5(1 \cdot 0) \\
13\end{array}$ & $\begin{array}{l}25 \\
0 \cdot 2 \\
0 \\
9 \cdot 7(1 \cdot 5) \\
11\end{array}$ & $\begin{array}{l}20 \\
0 \cdot 4 \\
0 \\
9 \cdot 9(1 \cdot 98) \\
6\end{array}$ \\
\hline
\end{tabular}


tween the patients who have survived and those who died in any of the arterial gas measurements or pulmonary function tests performed before or immediately after treatment. Similarly there were no differences between the two groups in the mean ages at the time of thoracoplasty or the intervals between thoracoplasty and starting assisted ventilation.

The two patients who did not comply adequately with treatment failed to maintain early improvements in their arterial blood gases. Their pretreatment $\mathrm{Po}_{2}$ values were $7.9 \mathrm{kPa}$ and $6.7 \mathrm{kPa}$, improving to $12.6 \mathrm{kPa}$ and $9.6 \mathrm{kPa}$ respectively at the initial post-treatment point, but then falling to mean values of $7.6 \mathrm{kPa}$ and $7 \cdot 4 \mathrm{kPa}$ over the subsequent period before death. Similar transient improvements were seen in their $\mathrm{PCO}_{2}$ values.

The seven patients who developed progressive cor pulmonale despite assisted ventilation tended to have lower mean $\mathrm{Po}_{2}$ values than the rest both before treatment $(6.53 \mathrm{v}$ $7 \cdot 28 \mathrm{kPa})$ and immediately after starting treatment $(8.5 v 10.1 \mathrm{kPa})$, although these differences were not significant. There were no significant differences between the two groups in the pulmonary function test values with no difference in degree of airflow obstruction.

\section{Discussion}

The total number of thoracoplasties performed for tuberculosis is not known. Phillips et al estimated that there may have been 30000 such operations carried out in the UK in the 1950s. ${ }^{11}$ They also showed that cardiorespiratory failure was common, and that the percentage mortality from it by decade since 1951 had steadily increased as the patients became older. If these data are extrapolated to the present day, then it is likely that over 17000 such patients are still alive of which $5-10 \%$ are likely to die of cardiorespiratory failure in the 1990 s if untreated.

Several studies have demonstrated the benefits of domiciliary ventilation in the management of patients with chronic ventilatory failure complicating neuromuscular and chest wall disorders including those who have undergone thoracoplasty. ${ }^{1219}$ The use of assisted ventilation in these patients leads, not only to physiological improvements and increased survival, but also to improvements in quality of life. ${ }^{20}$ Many of these studies, however, have reported mixed patient groups and several refer to the results of tracheostomy IPPV which may differ from non-invasive assisted ventilation of thoracoplasty patients. These studies suggest that patients with scoliosis or non-progressive neuromuscular disorders have the best survival and those with thoracoplasty or progressive neuromuscular disorders do less well. ${ }^{21}$ It is thought that the reduced survival in thoracoplasty patients is related to the associated airflow obstruction which is often severe. Sawicka et $a l^{22}$ reported the successful use of intermittent negative pressure ventilation in 10 patients with a thoracoplasty but the follow up data were limited. Leger et $a l^{23}$ reported the results of NIPPV for a mixed group of patients including 88 with what were described as mutilating sequelae of tuberculosis. Detailed physiological results were not available in this report but the one, three, and four year survival rates for the patients with tuberculosis were $86 \%, 75 \%$, and $71 \%$, respectively, which are close to the figures reported in this study.

The rapid and sustained improvement in daytime arterial $\mathrm{PO}_{2}$ and $\mathrm{PCO}_{2}$ seen in our patients has been seen in other patient groups treated with assisted ventilation. ${ }^{13171824}$ The possible mechanisms for this improvement include the recovery of respiratory centre sensitivity to carbon dioxide, relief of respiratory muscle fatigue, improvement in chest wall compliance, and relief of sleep deprivation..$^{25-28}$ The improvement in vital capacity which was seen shortly after starting assisted ventilation has also been seen in patients with scoliosis and chronic airflow limitation ${ }^{29} 30$ and may reflect improved chest wall compliance or result from the increased respiratory muscle strength which was observed in the present study.

Our patients used two different methods of assisted ventilation. Cuirass ventilation is a form of negative pressure ventilation which in our patients did not usually have a patient trigger. It occasionally precipitates upper airways obstruction which may compromise its effectiveness, ${ }^{31}$ but this problem was not seen in our patients. Cuirass ventilators are, at present, more reliable and cheaper than nasal ventilators. Nasal IPPV does not usually precipitate upper airways obstruction but periods of nocturnal oxygen desaturation do occur - often as a result of air leaks through the mouth. This was not a major problem in our patients. Six patients had to change from cuirass ventilation to NIPPV although only four of these were because of unsatisfactory ventilation. This study does not address the differences between the two methods in detail.

The optimal time for intervention in thoracoplasty patients with chronic stable ventilatory failure is uncertain. In practice the compliance of such patients with assisted ventilation is greater if the patient is symptomatic with, for example, dyspnoea and fatigue prior to treatment. Many patients, however, are relatively asymptomatic until shortly before their respiratory failure worsens. ${ }^{1122}$ It is probably important to start treatment before pulmonary hypertension develops since this may not be reversible and may be an important determinant of subsequent survival.

This study has confirmed the effectiveness of long term domiciliary assisted ventilation for thoracoplasty patients in respiratory failure. Patients who have had a thoracoplasty should be regularly reviewed by respiratory physicians and considered for assisted ventilation if there is evidence of ventilatory failure.

1 Cournand A, Richards DW. Pulmonary insufficiency. II. The effects of various types of collapse therapy on cardiopulmonary function. Am Rev Tuberc 1941;44:123-72.

2 Landis FB, Weisel W. Comparative study of pulmonary function loss: thoracoplasty versus small resection in surfunction loss: thoracoplasty versus small resection in

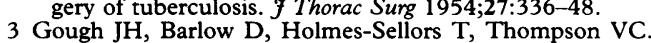
The results of thoracoplasty in the treatment of pulmonary The results of thoracoplasty in the treat
tuberculosis. Thorax 1957;12:241-52.

4 Loynes RD. Scoliosis after thoracoplasty. 7 Bone foint Surg (Br) 1972;54:484-98. 
5 Lancaster JF, Tomashefski JF. Tuberculosis - a cause of emphysema. Am Rev Respir Dis 1962;87:435-7.

6 Hallet WY, Martin CJ. The diffuse obstructive pulmonary syndrome in a tuberculosis sanatorium.(1) Etiologic factors. Ann Intern Med 1961;54:1146-55.

7 Phillips MS, Miller MR, Kinnear WJM, Gough SE, Shneerson JM. Importance of airflow obstruction after thoracoplasty. Thorax 1987;42:348-52.

thoracoplasty. Thorax 1987;42:348-52.
8 Quanjer PH. Standardised lung function testing. Eur Bull Physiopathol Respir 1983;19(Suppl 5):1-95.

9 Brown L, Kinnear WJM, Sargeant KA, Shneerson JM Artificial ventilation by external negative pressure: a method for making cuirass shells. Physiotherapy 1985;71: 181-3.

10 Kinnear WJM, Shneerson JM. The Newmarket pump: a new pump for external negative pressure ventilation. Thorax 1985;40:677-81.

11 Phillips MS, Kinnear WJM, Shneerson JM. Late sequelae of pulmonary tuberculosis treated by thoracoplasty. Thorax 1987;42:445-51.

12 Heckmatt JZ, Loh L, Dubowitz V. Night-time nasal ventilation in neuromuscular disease. Lancet 1990;335:579-82.

13 Goldstein RS, Molotiu N, Skrastins R, Lone S, De Rosie $\mathrm{J}$, Contreras $\mathrm{M}$, et al. Reversal of sleep induced hypoventilation and chronic respiratory failure by nocturnal negative ventilation in patients with restrictive ventilatory impairment. Am Rev Respir Dis 1987;135:1049-55.

14 Hoeppner VH, Cockroft DW, Dosman JA, Cotton DJ. Nighttime ventilation improves respiratory failure in secondary kyphoscoliosis. Am Rev Respir Dis 1984;129:240-3

15 Hill NS, Eveloff SE, Carlisle CC, Goff SG. Efficacy of noctural nasal ventilation in patients with restrictive thoracic disease. Am Rev Respir Dis 1992;145:365-71.

16 Jackson M, Kinnear W, King M, Hockley S, Shneerson J. The effects of five years of nocturnal cuirass-assisted ventilation in chest wall disease. Eur Respir $\mathcal{f} 1993 ; 6$ : ventilation.

17 Ellis ER, Grunstein RR, Chan S, Bye TP, Sullivan CE. Noninvasive ventilatory support during sleep improves respiratory failure in kyphoscoliosis. Chest 1988;94:811-5.

18 Carroll N, Branthwaite MA. Control of nocturnal hypoventilation by nasal intermittent positive pressure ventilation. Thorax 1988;43:349-53.

19 Leger P, Robert D, Langevin B, Guez A. Chest wall deformities due to idiopathic kyphoscoliosis or sequelae of tuberculosis. Eur Respir Rev 1992;2:10,362-8.

20 Sawicka EH, Loh L, Branthwaite MA. Domiciliary ventilatory support: an analysis of outcome. Thorax 1988;43: $31-5$.

21 Robert D, Gerard M, Leger P, Buffat J, Jennequin J, Holzapfel $\mathrm{L}$, et al. La ventilation mecanique a domicile definitive par tracheotomie de l'insufficient respiratoire chronique. Rev Fr Mal Respir 1983;11:923-36.

22 Sawicka EH, Branthwaite MA, Spencer GT. Respiratory failure after thoracoplasty: treatment by intermitten negative-pressure ventilation. Thorax 1983;38:433-5.

23 Leger P, Bedicam JM, Cornette A, Degat OR, Robert D, Polu JM, et al. Long term follow up of severe chronic insufficiency patients $(\mathrm{n}=373)$ treated by home nocturnal nasal IPPV. Am Rev Respir Dis 1991;143:A73.

24 Kinnear W, Hockley S, Harvey J, Shneerson J. The effects of one year of noctural cuirass-assisted ventilation in chest wall disease. Eur Respir 7 1988;1:204-8.

25 Carrey Z, Gottfried SB, Levy RD. Ventilatory muscle support in respiratory failure with nasal positive pressure port in respiratory failure with
ventilation. Chest $1990 ; 97: 150-8$.

26 Bergofsky EH. Respiratory failure in disorders of the thoracic cage. Am Rev Respir Dis 1979;119:643-69.

27 Douglas NJ, White DP, Pickett CK, Weil JV, Zwillich CW. Respiration during sleep in normal man. Thorax 1982;37 840-4.

28 Cooper KR, Phillips BA. Effect of short term sleep loss on breathing. F Appl Physiol 1982;53:855-8.

29 Fulkerson WJ, Wilkins JK, Esbenshade AM, Eskind JB, Newman JH. Life threatening hypoventilation in kyphoscoliosis: successful treatment with a moulded body brace-ventilator. Am Rev Respir Dis 1984;129:185-7.

30 Cropp A, Dimarco AF. Effects of intermittent negative pressure ventilation on respiratory muscle function in presients with severe chronic obstructive pulmonary dispatients with severe chronic obstructive pulm
ease. Am Rev Respir Dis 1987;135:1056-61.

31 Peltier LF. Obstruction apnea in artificially hyperventilated subjects during sleep. $\mathcal{f}$ Appl Physiol 1953;5:614-8. 